Trauma Berufskrankh 2009 • 11[Suppl 1]:18-21 DOI 10.1007/s10039-008-1424-7

Online publiziert: 13. September 2008

(c) Springer Medizin Verlag 2008

\author{
S. Bartsch \\ Abteilung für Unfallchirurgie, Orthopädie Krankenhaus Bethel, Bückeburg
}

\title{
Basisversorgung im Traumanetzwerk
}

1/4 aller Arbeitsunfähigkeitsjahre werden durch Verletzungen und Vergiftungen verursacht.

Andererseits hat die Anzahl an Krankenhausbetten von 1991-2004 um 20\% abgenommen. Die Fallzahlen sind jedoch um über $15 \%$ gestiegen [3]. Chirurgische Abteilungen sind vom Bettenabbau überproportional betroffen, auch der Mangel an qualifiziertem ärztlichem Nachwuchs trifft die chirurgischen Fächer besonders stark.

\section{Traumanetzwerk}

Aus den oben angeführen Punkten ergibt sich die Notwendigkeit einer flächendeckenden Traumaversorgung. Die folgenreichsten Verkehrsunfälle kommen weiterhin auf den außerörtlichen Straßen (ohne Autobahnen) vor. Sie machen 27,9\% aller Unfälle mit Personenschaden aus, sind aber für $62,7 \%$ der Verkehrstoten verantwortlich [3]. Nach wie vor ist insbesondere in den dünner besiedelten Flächenbundesländern die Erreichbarkeit eines Traumazentrums innerhalb von $30 \mathrm{~min}$ nicht gewährleistet, wie aus der Verteilung der Schwerpunkt- und maximalversorgenden Kliniken geschlossen werden kann (http://www.dgu-traumanetzwerk. de/traumanetzwerk/imagemaps/dgu/index.html).

Auch die Qualität der Schwerverletztenversorgung in Deutschland ist inhomogen, mit regional unterschiedlichen Versterbensraten [4]. Neben dem Problem der 30-min-Erreichbarkeit bestehen teilweise gravierende Unterschiede in der strukturellen und personellen Ausstattung und der Organisation der an der Traumaversorgung beteiligten Kranken- häuser, die zu Abweichungen in der Behandlungsqualität, auch zwischen universitären Traumazentren, führen $[1,4,5]$.

Die Initiative Traumanetzwerk der DGU (deutsche Gesellschaft für Unfallchirurgie) hat ein Organisationskonzept für eine qualitätsgesicherte flächendeckende Versorgung von Schwerverletzten entwickelt. Die Voraussetzungen und Qualitätsstandards für die Ausstattung und die Organisation der mit der Schwerverletztenversorgung betrauten Kliniken und eines regionalen Traumanetzwerks wurden im Weißbuch Schwerverletztenversorgung veröffentlicht [2]. Demnach soll die Fläche der Bundesrepublik Deutschland von regionalen Traumanetzwerken abgedeckt werden. Die beteiligten Kliniken werden in 3 Versorgungsstufen eingeteilt, mit unterschiedlichen Anforderungen bezüglich der vorzuhaltenden Fachabteilungen, strukturellen und personellen Ausstattung. Es werden überregionale und regionale Traumazentren sowie Einrichtungen der Basisversorgung von Schwerverletzten unterschieden. Innerhalb der regionalen Traumanetzwerke wird das Zusammenspiel der Kliniken unterschiedlicher Versorgungsniveaus unter Berücksichtigung regionaler Besonderheiten geregelt. Ziel ist es, dass jeder Schwerverletzte innerhalb von 30 min den Schockraum einer geeigneten Klinik erreicht. Innerhalb des regionalen Netzwerks sollen verbindliche, leitliniengerechte Verabredungen getroffen werden. Diese betreffen:

- Definierte Kriterien zur Aufnahme über den Schockraum eines Traumazentrums oder einer Einrichtung der Basisversorgung 
- Einbindung der präklinischen Rettungsdienste

- Einheitliches Schockraummanagement

- Formulierung standardisierter Behandlungsabläufe und Definition der Verlegungskriterien in ein übergeordnetes Traumazentrum

- Garantierte Patientenübernahme durch ein Traumazentrum

- Eindeutige und verlässliche Kommunikationswege (Traumatelefon, Bildund Befundtransfer)

- Fortbildung, Vereinheitlichung des Ausbildungsstandes

- Qualitätssicherung

\section{Anforderungen an Einrichtungen der chirurgischen Basisversorgung von Schwerverletzten}

Laut dem Weißbuch Schwerverletztenversorgung der DGU [2] liegt die Aufgabe der Einrichtungen der chirurgischen Basisversorgung in der Sicherstellung der Flächendeckung der akuten Versorgung Schwerverletzter innerhalb von $30 \mathrm{~min}$ :

- Verletzungen der Körperhöhlen, des Stamms, der Extremitäten und lebensbedrohliche Blutungen werden erkannt und behandelt.

- Notthorakotomien, Notlaparotomien und externe Beckenstabilisierungen müssen jederzeit durchführbar sein.

- Schwere Extremitätenverletzungen werden entsprechend der DamageControl-Strategie versorgt.

Die grundsätzliche Aufgabe besteht in der Herstellung der Transportfähigkeit für die Weiterverlegung in ein regionales oder überregionales Traumazentrum.

In Einrichtungen der Basisversorgung müssen laut Weißbuch 24-stündig verfügbar sein:

- Facharzt für Orthopädie/Unfallchirurgie mit Zusatzweiterbildung Spezielle Unfallchirurgie

- Facharzt für Viszeralchirurgie oder Allgemeine Chirurgie

- Facharzt für Anästhesiologie

- Bereitschaft der Notaufnahme für die Akutversorgung Schwerverletzter

- Operationsbereitschaft für Notfälle

- Räumliche, apparative und personelle Ausstattung zur notfallmäßigen

Trauma Berufskrankh 2009 · 11[Suppl 1]:18-21 DOI 10.1007/s10039-008-1424-7

(c) Springer Medizin Verlag 2008

\section{S. Bartsch \\ Basisversorgung im Traumanetzwerk}

\section{Zusammenfassung}

Die Qualität der Schwerverletztenversorgung in Deutschland ist inhomogen, mit regional unterschiedlichen Versterbensraten. Die Initiative Traumanetzwerk der DGU (deutsche Gesellschaft für Unfallchirurgie) hat ein Organisationskonzept für eine qualitätsgesicherte flächendeckende Versorgung von Schwerverletzten entwickelt, das im Weißbuch Schwerverletztenversorgung erläutert ist. Demnach soll die Fläche der Bundesrepublik Deutschland von regionalen Traumanetzwerken abgedeckt werden, wobei 3 Versorgungsstufen unterschieden werden: überregionale und regionale Traumazentren sowie Einrichtungen der Basisversorgung. Ziel ist letztendlich, dass jeder Schwerverletzte innerhalb von 30 min den Schockraum einer geeigneten Klinik erreicht. Einrichtungen der Basisversorgung im Traumanetzwerk sichern die flächendeckende kompetente Erstversorgung Schwerverletzter.

\section{Schlüsselwörter}

Deutschland · Schwerverletztenversorgung . Deutsche Gesellschaft für Unfallchirurgie . Initiative Traumanetzwerk · Weißbuch Schwerverletztenversorgung

\section{Basic medical care in a trauma network}

\section{Abstract}

The quality of care for serious injuries in Germany is not standard and regional differences in mortality rates can be seen. The initiative undertaken by the DGU, the German Society for Accident Surgery, is an organizational concept aimed at providing standard, quality-assured care for serious injuries, as defined in the White Paper Treatment of Serious Injuries. According to this concept, the whole of Germany should be covered by regional trauma networks, providing three levels of treatment: nationwide and regional treatment in trauma centers, as well as treatment in cen- ters for basic medical care. The ultimate goal of this concept is that every seriously injured patient should reach the trauma room in a suitable hospital within $30 \mathrm{~min}$. Basic medical care centers within the trauma network ensure that competent primary care for seriously injured patients is available nationwide.

\section{Keywords}

Germany - Treatment of serious injuries . German Society for Accident Surgery .

"TraumaNetwork" initiative - White Paper on Treatment of Serious Injuries 
Tab. 1 Personelle Anforderungen an Einrichtungen der chirurgischen Basisversorgung

\begin{tabular}{|c|c|}
\hline \multirow[t]{4}{*}{$\begin{array}{l}\text { Basisteam für die Versorgung } \\
\text { eines Schwerverletzten }\end{array}$} & $\begin{array}{l}1 \text { Weiterbildungsassistent (Orthopädie/Unfallchirurgie, } \\
\text { Viszeralchirurgie oder Allgemeine Chirurgie) }\end{array}$ \\
\hline & 2 Pflegekräfte Chirurgie \\
\hline & 1 Pflegekraft Anästhesie \\
\hline & 1 MTRA (medizinisch-technisches Radiologiepersonal) \\
\hline \multirow[t]{5}{*}{ Hintergrunddienst } & $\begin{array}{l}\text { Facharzt für Orthopädie/Unfallchirurgie mit Zusatzweiterbildung } \\
\text { Spezielle Unfallchirurgie (Oberarzt) }\end{array}$ \\
\hline & Facharzt für Viszeralchirurgie oder Allgemeine Chirurgie (Oberarzt) \\
\hline & Facharzt für Anästhesiologie (Oberarzt) \\
\hline & Facharzt für Radiologie \\
\hline & 2 Operationspflegekräfte \\
\hline
\end{tabular}

Triage und Versorgung von Schwerverletzten

\section{Struktur}

Strukturell ist eine Notaufnahme mit Schockraum gefordert, die auch interdisziplinär z. B. mit der Abteilung für Innere Medizin betrieben werden kann. Neben dem Schockraum sind eine Intensivstation und die jederzeitige, kurzfristige Operationsmöglichkeit im Bereitschaftsdienst vorzuhalten.

\section{Personal}

Die personellen Anforderungen sind in - Tab. 1 aufgeführt.

\section{Organisatorisch und apparativ}

Es wird eine zeitgemäße Ausstattung der Klinik, des Schockraums, der Intensivstation und der Operationseinheit gefordert, mit Blutdepot, Labor, Beatmungsmöglichkeit, Defibrillator, Herz-Kreislauf-Monitoring, Notoperationssets (Thorakotomie, Laparotomie, Beckenstabilisierung, Bülau-Dränage, Endoskopie, Tracheotomie), unfallchirurgischen Instrumentarien, Fixateur externe, Schienen- und Extensionssystemen, Ultraschallgerät, konventioneller Röntgenanlage, CT, Bildwandler, Cell-Saver sowie Physiotherapie und Sozialdienst.

\section{Einrichtung (über-)regionaler Traumanetzwerke}

Die Einrichtung eines Traumanetzwerks geht von einem oder mehreren überregionalen Traumazentren aus und wird seitens des Arbeitskreises AKUT (Arbeitskreis zur Umsetzung Weißbuch/Traumanetzwerk) der DGU begleitet (http://www. dgu-traumanetzwerk.de). In regionalen Treffen aller beteiligungswilligen Unfallchirurgen werden die regionalen Besonderheiten erörtert und das Traumanetzwerk umgesetzt. Am Ende des Prozesses steht die Zertifizierung durch die DGU.

Im Traumanetzwerk Region Hannover, einem Zusammenschluss von $32 \mathrm{Kli}$ niken in Niedersachsen, geht die Initiative von den Unfallchirurgischen Kliniken der Medizinischen Hochschule (Prof. Dr. Krettek) und des Friederikenstifts (Prof. Dr. Lill) aus. In mehreren Treffen wurden die regionale und die überregionale Versorgungsstruktur analysiert. Dabei waren die Versorgungsprofile einzelner Kliniken und Unterregionen, unterschiedliche Trägerschaften, Beziehungen in Randregionen des Netzwerks und gewachsene Strukturen zu analysieren. Hilfreich bei der Bestandsaufnahme war die initiale Befragung aller (unfall-)chirurgischen Kliniken Niedersachsens (Hildebrand, persönliche Mitteilung). Am Ende des Prozesses stehen verbindliche Verabredungen bezüglich der Primärversorgung Schwerverletzter der Region und Verabredungen mit den Nachbarregionen.

Die im Traumanetzwerk obligaten regelmäßigen Treffen der Unfallchirurgen einer Region fördern den persönlichen Austausch. Die Institution Traumanetzwerk hilft auch im Diskussionsprozess der einzelnen leitenden Unfallchirurgen mit ihren Verwaltungen und Trägern, die aus unterschiedlichen Gründen nicht immer deckungsgleiche Interessen verfolgen. Das Traumanetzwerk kann den unfallchirurgischen Teamgeist stärken, schafft Gemeinsamkeiten und verleiht der Unfallchirurgie eine Stimme in der Region.
Dies wird in der Zukunft noch wichtiger werden.

Konkrete Verabredungen über Kommunikationswege (Traumatelefon) und Datenaustausch (Homepage, FTP-Server für gemeinsame Diskussion von Röntgen, CT-, MRT-Bildern) und die Abnahmegarantie durch die überregionalen Traumazentren haben sich in der Praxis bereits bewährt.

Aus Sicht der basisversorgenden Kliniken wird besonders die gemeinsame, regelmäßige Fortbildung im Traumanetzwerk begrüßt. Gemeinsame Standards in der Erstversorgung Schwerverletzter, wie ein einheitliches Schockraumprotokoll, werden auf der Grundlage der Leitlinien der DGU formuliert und über gemeinsame Fortbildungen in die Klinikmannschaften getragen. Fall- und Komplikationsdiskussionen finden nun nicht mehr nur klinikintern, sondern klinikübergreifend im Netzwerk statt, mit entsprechend größerem Erkenntnisgewinn. Die Möglichkeiten der Umsetzung gemeinsamer Fortbildungen, Schockraumkurse, Workshops oder Hospitationen in den Traumazentren sind im Traumanetzwerk vielfältig umsetzbar. Diese Angebote kann ein grundversorgendes Krankenhaus mit begrenzten personellen Ressourcen seinen Ärzten kaum zur Verfügung stellen. Das Internet bietet zusätzliche Möglichkeiten des Austausches. In der Summe wird das aktiv gelebte Traumanetzwerk die Versorgung Schwerverletzter verbessern und das Profil der Unfallchirurgie schärfen.

Es darf nicht vernachlässigt werden, was in den Krankenhäusern geschieht, die den Zertifizierungskriterien nicht genügen. Insbesondere die personellen Anforderungen des Weißbuches an Einrichtungen der Basisversorgung sind von nicht wenigen Krankenhäusern und $\mathrm{Ab}$ teilungen (noch) nicht zu erfüllen, die heute an der qualifizierten Versorgung Schwerverletzter teilnehmen und die die flächendeckende unfallchirurgische Versorgung in Deutschland gewährleisten. Auch in diesen Kliniken sind qualifizierte Kollegen seit Jahren kompetent tätig.

- Ist in allen Abteilungen, die heute die Erstversorgung Schwerverletzter durchführen, immer ein Spezieller Unfallchirurg $24 \mathrm{~h}$ verfügbar? 
- Wie verhält es sich mit Abteilungen, die, im Zeitalter des gewünschten $\mathrm{Zu}$ sammenwachsens der Gebiete, auch Spezielle Orthopäden beschäftigen?

- Wie sieht es mit erfahrenen Fachärzten für Chirurgie aus, die jahrelang schwerpunktmäßig unfallchirurgisch tätig waren?

Bei diesen Fragen sind Augenmaß und Gespür für regionale Besonderheiten gefragt. Das Traumanetzwerk sollte keine geschlossene Gesellschaft sein, sondern übergreifend auch die Unfallchirurgie in den grundversorgenden Krankenhäusern im Blick haben. Auch wenn es hier nicht täglich um die Versorgung Schwerverletzter geht, sollte die Kompetenz dafür jenseits des Traumanetzwerks nicht verloren gehen. Die zukünftigen Erfordernisse in der Alterstraumatologie können nur mit Hilfe einer ordentlichen Unfallchirurgie auch in nicht zertifizierten grundversorgenden Häusern bewältigt werden.

\section{Fazit}

Basisversorgung im Traumanetzwerk ist die Grundlage der flächendeckenden, kompetenten Erstversorgung Schwerverletzter, insbesondere in Flächenländern. Das (über-)regionale Traumanetzwerk definiert und koordiniert die Schwerverletztenversorgung einer Region auf der Grundlage der Kriterien des Weißbuch Schwerverletztenversorgung und der Leitlinien der DGU. Alle Beteiligten des Traumanetzwerks können von diesem Prozess profitieren. Standards und Verabredungen bezüglich der präklinischen Versorgung, des Schockraummanagements, der Kommunikationspfade, der Behandlungspfade, definierter Verlegungskriterien, der verlässlichen Übernahme Schwerverletzter durch Traumazentren, der gemeinsamen Fortbildung und Fehlerdiskussion sowie der Qualitätssicherung werden zweifellos die Versorgung Schwerverletzter verbessern. Die Traumanetzwerke repräsentieren unfallchirurgisches Profil in einer Region.

\section{Internetlinks}

- http://www.destatis.de (Homepage des Statistischen Bundesamts)

- http://www.dgu-online.de (Homepage der Deutschen Gesellschaft für Unfallchirurgie)

- http://www.dgu-traumanetzwerk.de (Traumanetzwerk)

- http://www.dgu-traumanetzwerk.de/ traumanetzwerk/imagemaps/dgu/index. html (Verteilung der Schwerpunkt- und maximalversogenden Kliniken)

\section{Korrespondenzadresse}

\section{Dr. S. Bartsch}

Abteilung für Unfallchirurgie, Orthopädie Krankenhaus Bethel,

Herminenstraße 12/13, 31675 Bückeburg s.bartsch@krankenhaus-bethel.de

Interessenkonflikt. Der korrespondierende Autor gibt an, dass kein Interessenkonflikt besteht.

\section{Literatur}

1. Biewener A, Aschenbrenner U, Rammelt S et al. (2004) Impact of helicopter transport and hospital level on mortality of polytrauma patients. J Trauma 56: 94-98

2. Deutsche Gesellschaft für Unfallchirurgie (2006) Weißbuch Schwerverletztenversorgung - Empfehlungen zur Struktur und Organisation von Einrichtungen zur Behandlung von Schwerverletzten in der Bundesrepublik Deutschland. DGU, Berlin, http://www.dgu-onlinde.de

3. Gesundheitsberichterstattung des Bundes (2006) Gesundheit in Deutschland. Berlin, Juli 2006, http://www.gbe-bund.de

4. Kühne CA, Ruchholtz S, Buschmann C et al. (2006) Initiative Traumanetzwerk DGU. Schwerverletztenversorgung in Deutschland - eine Standortbestimmung. Unfallchirurg 109: 357-366

5. Ruchholtz S, Kühne CA, Siebert H (2007) Das Traumanetzwerk der Deutschen Gesellschaft für Unfallchirurgie. Unfallchirurg 110: 373-380

6. Statistisches Bundesamt (Hrsg) (2007) Datenreport 2006. Zahlen und Fakten über die Bundesrepublik Deutschland. Statistisches Bundesamt, Wiesbaden, http://www.destatis.de

7. Statistisches Bundesamt (2004) Strassenverkehrsunfälle im Jahr. Statistisches Bundesamt, Wiesbaden, http://www.destatis.de 